\title{
An optically transparent ultra high speed LAN-ring employing OTDM
}

\author{
K. Bengi, G. Remšak, H.R. van As \\ Vienna University of Technology, \\ Institute of Communication Networks \\ Gusshausstrasse 25/388, A-1040 Vienna, Austria \\ Tel.: +43-1-58801-3518, Fax: +43-1-5870583, \\ email:kemal.bengi@tuwien.ac.at
}

\begin{abstract}
In this paper, an optically transparent $100 \mathrm{Gbit} / \mathrm{s}$ ring-LAN architecture based on the slotted ring protocol and the OTDM technique is presented. Optical transparency is achieved by using optical packets of constant duration which consist of variable bit rate payloads and headers with fixed data rate. Thus, the considered LAN is capable of providing multiple services for nodes with different transmission speeds.
\end{abstract}

\section{Keywords}

photonic networks, ring-LAN, OTDM

\section{INTRODUCTION}

In the recent past, Local Area Networks (LANs) with various network topologies and media access methods have been proposed. Nowadays, LANs must be capable of integrating multiple services like multimedia, videoconferencing, fast database access, high-performance interprocessor communications and many other high- bandwidth 
applications. For that reason, future networks have to provide data rates in the upper Gbit/s range on instantaneous demand with high overall channel efficiency and minimal latency, which can only be realized by photonic networks.

Currently, the utilization of the optical fiber bandwidth in high-speed shared medium LANs is limited to a few Gbit/s due to the electronic bottleneck in processing the optical signals in the network nodes. In consequence, the nodes of a conventional local area network communicate at a single low bit rate with each other, since the handling of the data packets is presently performed electronically using optoelectronic conversion at each node. In order to achieve aggregate data rates up to Tbit/s ranges optical processing methods have to be applied, thereby removing the conversion between the electrical and optical domain in the optical signal path.

A very promising technique in this context is optical time-division multiplexing (OTDM), which yields aggregate capacities beyond the reach of high-speed electronics. In general, OTDM (local area) networks provide interconnections with very high guaranteed bandwidth, low latency and broadcast capability resulting in bandwidth on demand with low access delay. In contrast to WDM networks, which divide fiber bandwidth into many optical wavebands carrying information at electronic rates $(\approx 2.5-40 \mathrm{Gbit} / \mathrm{s})$, OTDM networks use short pulses to transmit at very high bit rates (> 100Gbit/s) in a single waveband.

The main advantages of OTDM networks are in general:

- interconnection of ultra high-speed nodes and networks

- fast transmission of very large data blocks

- switching of large traffic volume

- flexible access to the medium

Thus, OTDM networks are suitable for satisfying the demand for mixed high-speed traffic. There are several theoretical and experimental investigations on OTDM bus networks to date ([Ellis, 1994], [Lucek, 1996], [Barry, 1996]).

In this paper, an optically transparent 100Gbit/s ring-LAN architecture based on the slotted ring mechanism and employing the OTDM technique is presented. Optical transparency is achieved by using optical packets of constant duration which consist of variable bit rate payloads and headers with fixed data rate. Furthermore, special 
OTDM nodes, at which multiple channels are multiplexed by OTDM, are integrated into the network.

\section{NETWORK ARCHITECTURE}

The proposed network serves nodes with different processing capabilities. While the optical medium operates at a line-rate of maximally $100 \mathrm{Gbit} / \mathrm{s}$, the user bandwidth requirements vary. The media access protocol of the LAN is based on the slotted ring technique with destination release and spatial slot reuse. Fixed-period slots carrying optical packets circulate permanently on the ring and can be empty or full. The ring nodes with traffic from attached stations can fill multiple slots in each ring revolution with their data packets. The choice of this protocol is due to its suitability for realtime traffic and for network transparency. Destination release is proposed since its use results in efficient channel utilization and an increase in ring capacity. In consequence, by allowing destination release and reuse of slots, the network throughput significantly exceeds the transmission rate of the medium. In contrast to alternative multiplexing methods such as WDM or bit-interleaved TDM ([Ellis, 1994]), the slotted TDM scheme enables sharing one fast medium by access nodes capable of operating at $100 \mathrm{Gbit} / \mathrm{s}$ rates. Thus, we can design a network architecture which simultaneously provides guaranteed bandwidth and random access bandwidth on demand services. Consequently, the provision of multiple services to a heterogeneous user population becomes feasible.

Figure 1 demonstrates the basic principle of operation of the considered ring-LAN. 


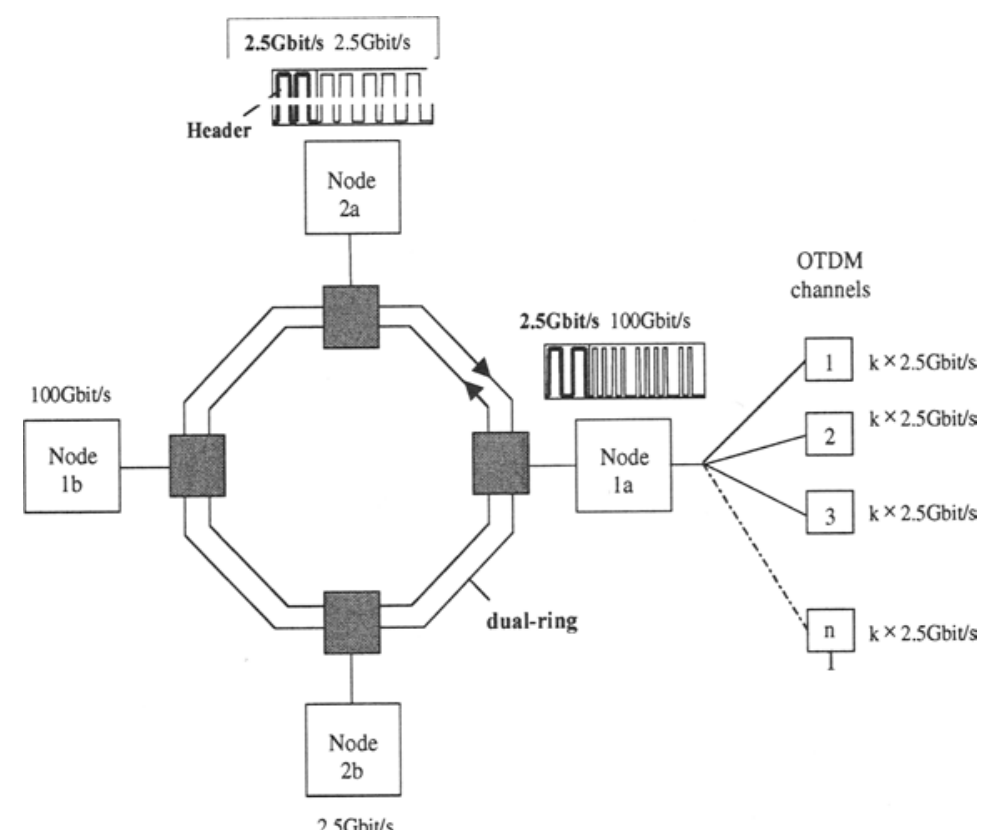

Figure 1 Basic configuration of the optical transparent ring-LAN.

As it can be seen in the figure, Node 1a has the capability of transmitting with an exemplary bit rate of $100 \mathrm{Gbit} / \mathrm{s}$ by optical time-division multiplexing of various local channels. These channels operate at $k$ multiples of the arbitrarily chosen standard data rate of $2.5 \mathrm{Gbit} / \mathrm{s}$. The header speed is uniformly maintained at $2.5 \mathrm{Gbit} / \mathrm{s}$ across the entire network. Since Node $1 \mathrm{~b}$ also represents an OTDM node equipped with OTDM demultiplexer and optical clock recovery facilities, it can properly process the corresponding 100Gbit/s signal sent from Node 1a. Similarly, the $2.5 \mathrm{Gbit} / \mathrm{s}$

conventional, i.e. only electronically operating, Node 2 a can communicate with the corresponding Node $2 \mathrm{~b}$ directly and straightforward. The data exchange between low bit rate nodes like Node 2a, 2b and OTDM nodes (Node 1a, 1b) is also easily feasible, since the optical clock associated with the OTDM demultiplexer may be adjusted to the appropriate repetition rate (in this case to $2.5 \mathrm{Gbit} / \mathrm{s}$ ) in order to extract the desired low bit rate signal. Naturally, the OTDM nodes have to know about the processing capabilities of their destination nodes, when they want to transmit packets to them. 
The slots for the packets to transmit have a fixed duration and consist of a payload and a header section. Moreover, they are separated by guard bands. For example, a network with the length of $1 \mathrm{~km}$ and consisting of 10 attached nodes and 40 slots yields a slot period of $125 \mathrm{~ns}$. The payload is to be transparent to bit rate and transmission format. The header contains source/destination addresses and control information (full/empty flag, monitor field, priority fields).

Due to the dual-ring configuration, the shortest path among the two fiber loops is chosen for effectively transmitting the optical packets to the specific destination.

The desired optical transparency is achieved by the provision of bit rate variable payloads and a header with fixed bit rate. Consequently, the optical packets consist of mixed rate data streams ([Cai, 1994]). Since the attached nodes operate at different speeds and the generation of optical signals with very high bit rates $(20-100 \mathrm{Gbit} / \mathrm{s})$ is performed by OTDM, we have to differentiate between the conventional electronic and the optical processing of the optical packets. Therefore, optically transparent photonic packet switching is to be used allowing payload bit rates to be independent of the header's data rate. Thus, only the header of each packet needs to be read by all the ring nodes, whereas the payload does not need to be perceived by all nodes. The bit rate of the payload is determined by the transmitting node, based on the capabilities of the corresponding receiving nodes. For that reason, different network nodes are capable of operating at different bit rates simultaneously. In consequence, this ensures that higher-speed nodes may be added to the network at a later date without requiring any changes in the lower-speed nodes currently operating in the network. On the other hand, this approach requires that each node knows about the bit rate capabilities of the other network nodes. This can be achieved either by using look-up tables for the achievable transmission speeds of the individual nodes on the ring or by polling each node before establishing a connection to that node. The lowest bit rate employed within the network could be $2.5 \mathrm{Gbit} / \mathrm{s}$ (corresponding to the STM16 format of SDH systems). In order to enable nodes to operate at very high bit rates like $100 \mathrm{Gbit} / \mathrm{s}$, OTDM is to be used. This can be done by multiplexing various channels of a specific node with respect to the processable bit rate of the destination node. If the destination nodes are not designed for operating at very high data rates, the corresponding OTDM source node may switch to an electronical transmission unit. 


\section{NODE DESIGN}

In order to provide optical transparency, the nodes detect only the headers operating at a fixed low bit rate and the payload of a packet is only read when it is destined to a specific node. Thus, an inexpensive node with a lower transmission speed does not need to process the optical time-division multiplexed signal ,rom an expensive node operating, for example, at $100 \mathrm{Gbit} / \mathrm{s}$. In the proposed network architecture, there are two main node configurations to be considered. Firstly, nodes operating at low bit rates up to 20Gbit/s and secondly the nodes using OTDM to achieve ultra high bit rates like $100 \mathrm{Gbit} / \mathrm{s}$, for which the proposed network is mainly designed.

The principle of the node's operation, which extends the scheme in [Cai, 1994] is demonstrated in Figure 2, where specifically the configuration for the OTDM node is depicted.

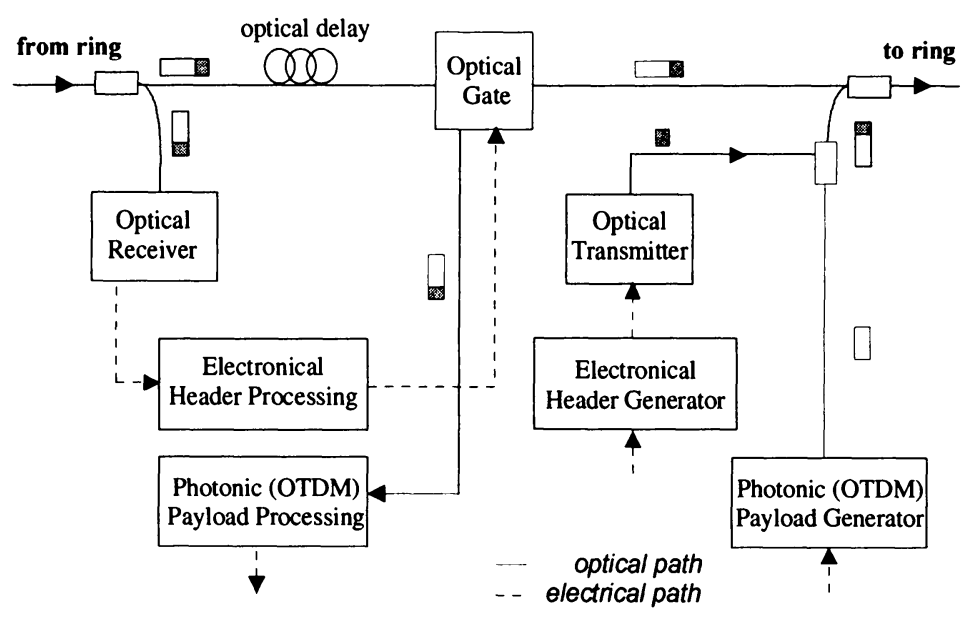

Figure 2 OTDM node configuration.

The block diagram for the low bit rate nodes operating electronically can be derived directly from the OTDM case. Let us consider the functionality of the high-speed node.

The input fiber coupler couples a fraction (commonly 10/90) of the light from the incoming optical slot. An optical delay line delays the slot while the header is received and processed. For the dimensioning of the optical delay line also the delays at the transmitter side of the node has to be taken into account. The headers (e.g. $2.5 \mathrm{Gbit} / \mathrm{s}$ ) of all slots that pass the node are detected by the optical receiver and 
processed by the electronical header processing block. Then, the electronic control unit triggers the further operations of the node components depending on whether the slots are addressed to the corresponding node or not. Particularly, it triggers also the transmitter part, which is not explicitly indicated in Figure 2.

In general, three possible situations could occur:

(1) If an incoming slot is not addressed to the node, it simply propagates through the node (i.e the optical gate is set to the "on" state).

(2) If the destination address of an incoming slot matches the address of the node, the payload processing block reads the payload data (using an passive coupler integrated in the optical gate block) and removes it from the ring according to the destination release rule, while the optical gate is set to the "off" state, thus blocking the transmission of the slot. Simultaneously, the node's transmitter section generates either an empty or a full slot depending on the existence of new data to be transmitted. The generation of an empty slot is straightforward since it does not require photonic operations. A new filled slot has to be created by independently generating the header (via the electronical header generator) and the payload. In order to obtain the payload, various local channels of the OTDM node have to be optically multiplexed via an OTDM multiplexer to achieve the desired very high transmission speeds. Then, the resulting OTDM payload signal is appended to the header. After combining header and high-speed payload, the new slot is put onto the ring via the output fiber coupler. Concerning the receiver part of the node, it has to be noted that the payload processing block consists of typical OTDM components such as the OTDM demultiplexer and the optical clock recovery unit. In the case of the low bit rate nodes, the payload is processed electronically. Thus, the node requires an additional O/E conversion between the optical gate and the payload processing unit.

(3) If an incoming slot is empty, indicated by an empty flag or by continuous wave light, the node inserts a new packet onto the ring by using the OTDM multiplexer, while the transmission of the empty slot is blocked through the optical gate. In the case of low bit rate nodes and indicating an empty slot by a cw-signal there is also the possibility to modulate the cw-signal by the optical gate to insert a new packet. 
54 Part Three: Optical technologies for local and access networks

As optical gate within the node a semiconductor optical amplifier (SOA) could be employed to gate or block packets, modulate packets and providing optical gain to overcome losses in the system.

\section{CONCLUSION}

In order to achieve ultra high-speed LANs fulfilling the requirements of highbandwidth applications, an optically transparent ring network with a maximum linerate of $100 \mathrm{Gbit} / \mathrm{s}$ employing the OTDM technique has been presented. The considered LAN is based on the slotted ring mechanism with destination release for its advantages in terms of optical transparency, bandwidth efficiency and transmission delays. Due to the concept of bit rate variable payloads, expensive nodes with high bit rate capabilities may coexist with low-cost nodes operating at low data rates. The main advantage of the proposed architecture consists in the possibility of supplying a heterogeneous user population with guaranteed bandwidth and bandwidth on demand services. Currently, a specific MAC (Medium Access Control) protocol for the introduced LAN architecture is being developed in order to guarantee fairness for the attached nodes of the ring-LAN.

\section{REFERENCES}

Ellis A.D., Widdowson T. et. al. (1994) Three-node, 40Gbit/s OTDM network experiment using electro-optic switches. Electronics Letters, 16, 1333-34.

Lucek J.K., Gunning P. et. al. (1996) 40Gbit/s Optical TDMA LAN. ECOC'96(Oslo) - Proceedings , 5.45-5.48.

Gunning P., Lucek J.K. et. al. (1997) 40Gbit/s Optical-TDMA LAN Over 300 Metres Installed Blown Fibre. ECOC'97(Edinburgh) - Proceedings, 61-64 .

Barry R.A., Chan V. et. al. (1996) All-Optical Network Consortium - Ultrafast TDM Networks. IEEE Journal on Selected Areas in Communication, 5, 999-1013.

Cai Y. et. al. (1994) Demonstration of Photonic Packet-Switched Ring Network with Optically Transparent Nodes. IEEE Photonics Technology Letters, 9, 1139-41. 
Chan C.K. et. al. (1995) Experimental demonstration of a high-speed all-optical tunable-channel multi-access (TCMA) network. Globecom'95, 1761-65.

\section{BIOGRAPHY}

Kemal Bengi received his Dipl.-Ing. degree in Electrical Engineering from the University of Erlangen (Germany) in 1997. Since May 1997 he is research assistant working towards his Ph.D. degree at the Institute of Communication Networks, Vienna University of Technology. His research interests include photonic networks, MAC protocol design and performance evaluation.

Günter Remšak received his Dipl.-Ing. degree in Technical Physics from the Vienna University of Technology in 1996. Since March 1997 he is research assistant working towards his $\mathrm{Ph} . \mathrm{D}$. degree at the Institute of Communication Networks, Vienna University of Technology. His research interests include photonic networks, optical transmission and performance evaluation.

Harmen R. van As received his Dipl.-Ing. degree in Electrical Engineering from the ETH Zurich and the Ph.D. degree from the University of Siegen (Germany). Since March 1996 he is full-professor and head of the Institute of Communication Networks, Vienna University of Technology. 